\title{
Structure-based assessment and network analysis of targeting 14-3-3 proteins in prostate cancer
}

\author{
Alex Root ${ }^{1}$ (D) Azadeh Beizaei ${ }^{2}$ and H. Alexander Ebhardt ${ }^{2^{*}}$ (D)
}

\begin{abstract}
Developing combination therapy for castrate-resistant prostate cancer (CRPC) may require exploiting new drug targets outside androgen receptor and PI3K / AKT / mTOR signal transduction pathways implicated in prostate cancer (PCa) progression. One such possible new target is YWHAZ of the 14-3-3 protein family as this gene has prognostic significance for metastatic CRPC patients. However, there are no small molecules targeting YWHAZ commercially available. Hence, we explored whether the small molecule BV02 targeting another 14-3-3 protein family member SFN also binds to YWHAZ. Using advanced docking algorithms we find that BV02 docks many other 14-3-3 family members. In addition, the amphipathic groove where drug binding occurs also has a high binding affinity for other drugs used to treat PCa such as docetaxel. The proteome of metastatic PCa models (LNCaP clone FGC and PC-3) was perturbed as a result of BV02 treatment. Through data integration of three proteomics data sets we found that BV02 modulates numerous protein-protein interactions involving 14-3-3 proteins in our PCa models.
\end{abstract}

Keywords: BV02, Structure-based drug design, MM-GBSA, Docetaxel, 14-3-3 protein family, YWHAZ

Metastatic castrate-resistant prostate cancer (mCRPC) affects approximately 1 in 30 men in the United States and there is an unmet need for effective therapy. Many mCRPC patients receive the chemotherapy drug docetaxel which prevents microtubule depolymerization thus arresting cell division. Advances in drug design and development lead to new compounds targeting the androgen receptor (AR) as a treatment option for mCRPC [1]. Despite these advances, 29,430 men will die of prostate cancer (PCa) in the U.S.A in 2018 [2]. A simulation study based on survival data of $2353 \mathrm{PCa}$ patients concluded that a two-drug combination therapy is unlikely to cure mCRPC patients [3]. Hence, searching for higher-order drug combinations targeting pathways in addition to $\mathrm{PI} 3 \mathrm{~K} / \mathrm{AKT} / \mathrm{mTOR}$ is warranted. One source for drug targets is recurrently amplified genomic regions. In approximately $30 \%$ of patients with metastatic $\mathrm{PCa}$ regions of chromosome $8 \mathrm{q}$ are amplified which includes genes like MYC, NCOA2, and YWHAZ. The latter protein was found

\footnotetext{
* Correspondence: h.a.ebhardt@ucd.ie

${ }^{2}$ Systems Biology Ireland, University College Dublin, Belfield, Dublin 4, Ireland Full list of author information is available at the end of the article
}

to be upregulated as a function of pharmacological intervention [4]. Taken together, these studies suggest YWHAZ is a potential drug target for PCa therapy. Binding a large number of proteins makes the 14-3-3 family an attractive tool for modulating protein activity, and thereby controlling multiple signal transduction pathways suggesting its role as universal regulators [5].

A nonpeptidic small molecule inhibitor of SFN termed BV02 induces apoptosis in chronic myeloid leukaemia models [6]. Subsequent NMR studies determined that BV02 is a labile compound in aqueous solution and the phthalimide derivative of BV02 termed BV02_9 serves as bioactive molecule [7]. Given that YWHAZ has close homology with SFN we hypothesized that BV02 also inhibits YWHAZ.

\section{Results and discussion}

Assessment of binding affinities of multiple compounds with 14-3-3 family members using molecular mechanics To confirm our hypothesis, we used structure-based analysis to assess the binding affinity of BV02 to YWHAZ. To compare the docking of BV02 and other 
small molecules between all seven 14-3-3 protein family members we used the Molecular Mechanics GeneralizedBorn Solvent Accessibility method, implemented within the Schrodinger Software Suite. The resulting free energy values $(\Delta G)$ allow for direct comparison within our seven protein - six small molecule matrix. In case of BV02 binding to all seven 14-3-3 protein family members with examples of docking shown in Fig. 1a and b. Differential $\Delta G$ were observed between protein family members with $\Delta \mathrm{G}$ values ranging from $-26.7 \mathrm{kcal} / \mathrm{mol}$ in case of YWHAB to - $53.0 \mathrm{kcal} / \mathrm{mol}$ for YWHAE (Fig. 1d, Table 1). The target protein SFN has a binding affinity of $-47.8 \mathrm{kcal} / \mathrm{mol}$ suggesting that BV02 is a promiscuous binder. The bioactive form BV02_9 has a smaller dynamic range for $\Delta \mathrm{G}$ compared to $\mathrm{BV} 02$ with values ranging from $25.1 \mathrm{kcal} / \mathrm{mol}$ (YWHAB) to $-39.7 \mathrm{kcal} / \mathrm{mol}$ (YWHAE) suggesting less specificity towards anyone 14-3-3 protein family member.

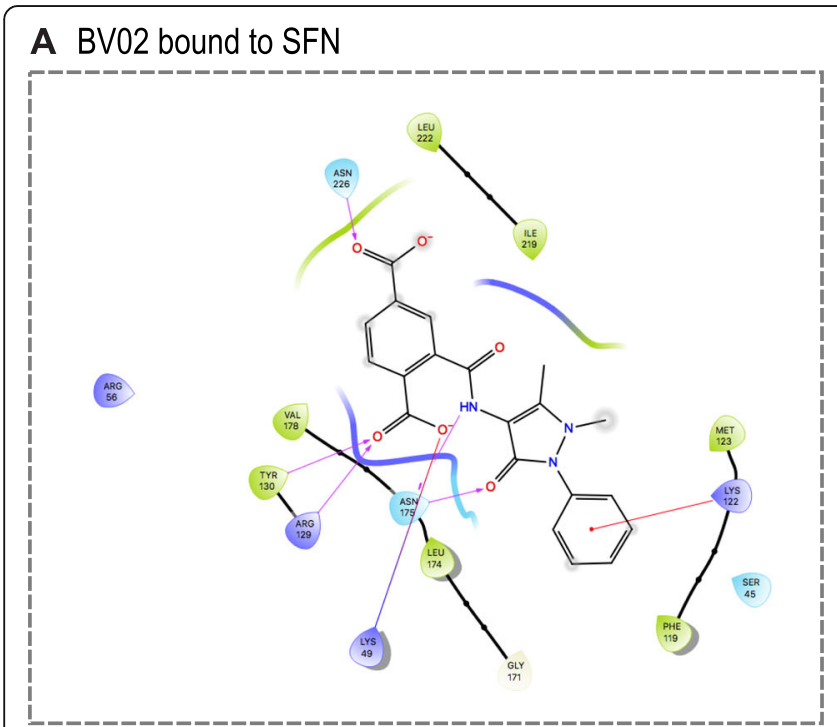

\section{B BV02 bound to YWHAZ}

C Legend for panels $\mathrm{A}, \mathrm{B}$, and $\mathrm{E}$
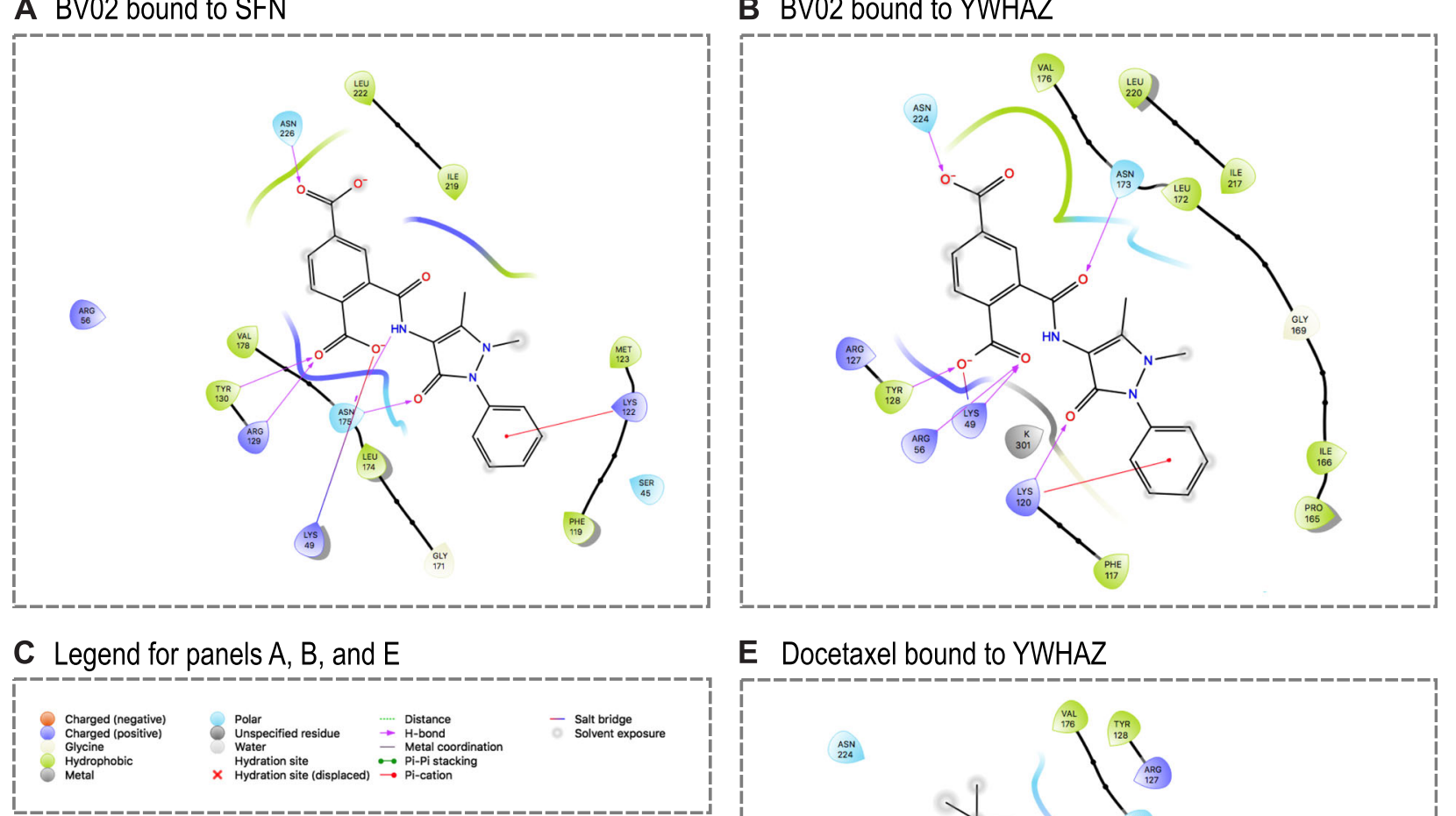

E Docetaxel bound to YWHAZ

\section{D $\Delta G$ values per small molecule inhibitor}
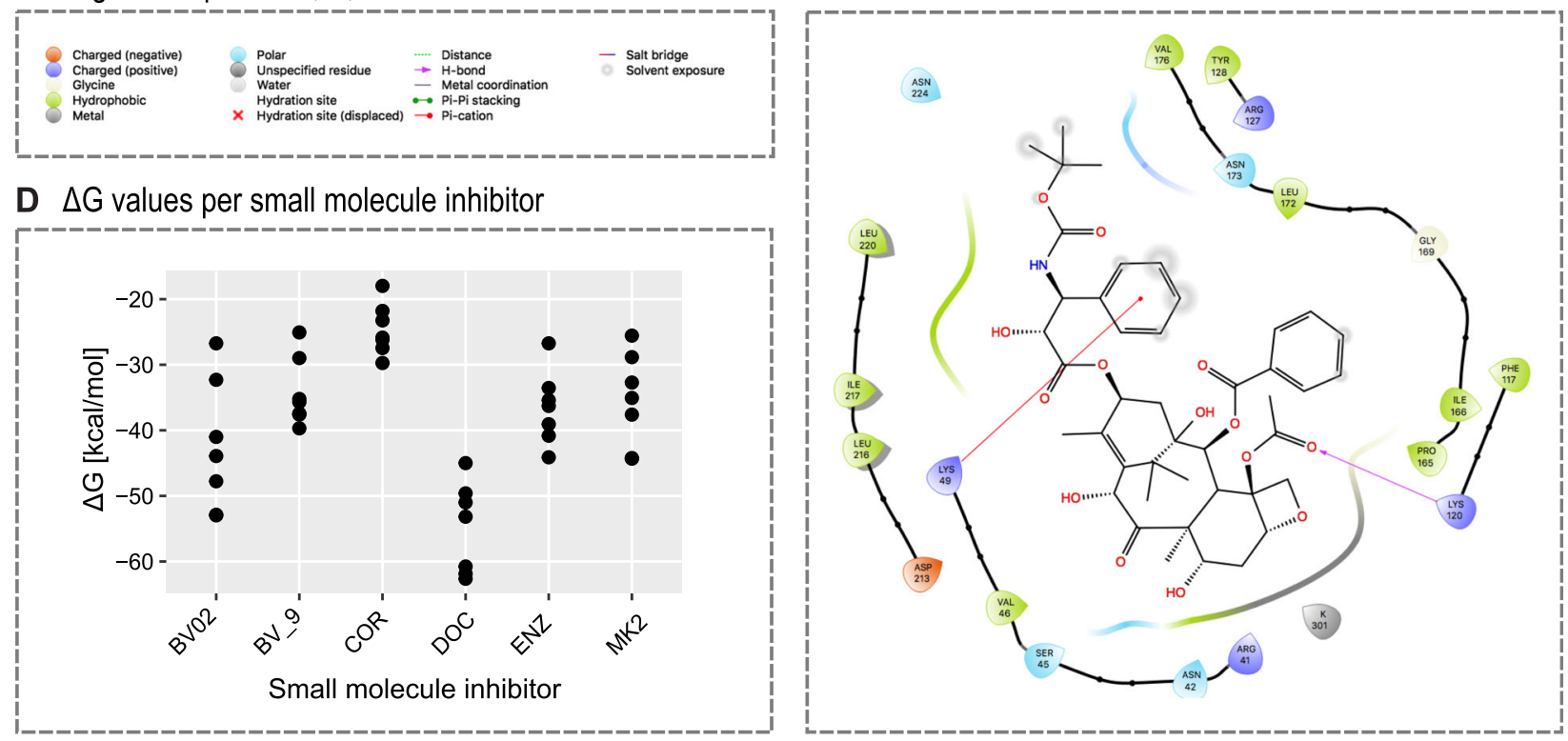

Fig. 1 Results of docking studies. In color on the outside are amino acid residues while small molecules are represented with their chemical structure. $\mathrm{H}$-bonds are represented as solid purple lines while pi-cation interactions are red. a Ligand interaction diagram between BV02 and SFN coordinated by H-bonds and pi-cation interaction. Several residues important for the protein-drug interaction include Lys-49, Arg-129, and Tyr-130. b Ligand interaction diagram between BV02 and YWHAZ involves different residues compared to SFN, e.g. Lys-49. c Figure legend for panels A, B, and E. d Free energy of binding $(\Delta \mathrm{G})$ for all seven 14-3-3 protein family member plotted for each small molecule inhibitors (BV02; BV02_9; COR: corannulene; DOC: docetaxel; ENZ: enzalutamide; MK2: MK2206). e Although docetaxel is only coordinated by a H-bond and a pi-cation interaction, the molecule fits squarely into the amphipathic binding groove resulting in very low $\Delta G$ values 
Table 1 Docking scores and binding free energy estimation with all 14-3-3 family members

\begin{tabular}{|c|c|c|}
\hline Protein & Drug & MMGBSA $\triangle \mathrm{G}$ Bind \\
\hline$\overline{S F N}$ & Docetaxel & -50.995 \\
\hline SFN & BV02 & -47.767 \\
\hline SFN & BV02_9 & -37.437 \\
\hline SFN & Enzalutamide & -35.406 \\
\hline SFN & Corannulene & -27.451 \\
\hline SFN & MK2206 & -25.558 \\
\hline YHWAQ & Docetaxel & -49.609 \\
\hline YHWAQ & MK2206 & -44.319 \\
\hline YHWAQ & BV02 & -41.003 \\
\hline YHWAQ & Enzalutamide & -36.277 \\
\hline YHWAQ & BV02_9 & -28.979 \\
\hline YHWAQ & Corannulene & -26.181 \\
\hline YWHAB & Docetaxel & -44.997 \\
\hline YWHAB & MK2206 & -35.065 \\
\hline YWHAB & Enzalutamide & -33.543 \\
\hline YWHAB & BV02 & -26.734 \\
\hline YWHAB & BV02_9 & -25.072 \\
\hline YWHAB & Corannulene & -21.792 \\
\hline YWHAE & Docetaxel & -60.766 \\
\hline YWHAE & BV02 & -52.966 \\
\hline YWHAE & MK2206 & -44.228 \\
\hline YWHAE & Enzalutamide & -44.112 \\
\hline YWHAE & BV02_9 & -39.695 \\
\hline YWHAE & Corannulene & -17.976 \\
\hline YWHAG & Docetaxel & -61.867 \\
\hline YWHAG & Enzalutamide & -40.822 \\
\hline YWHAG & MK2206 & -37.603 \\
\hline YWHAG & BV02_9 & -35.213 \\
\hline YWHAG & BV02 & -32.296 \\
\hline YWHAG & Corannulene & -25.861 \\
\hline YWHAH & Docetaxel & -53.17 \\
\hline YWHAH & BV02 & -52.878 \\
\hline YWHAH & BV02_9 & -35.691 \\
\hline YWHAH & Corannulene & -29.735 \\
\hline YWHAH & MK2206 & -28.84 \\
\hline YWHAH & Enzalutamide & -26.734 \\
\hline YWHAZ & Docetaxel & -62.634 \\
\hline YWHAZ & BV02 & -43.919 \\
\hline YWHAZ & Enzalutamide & -39.064 \\
\hline YWHAZ & BV02_9 & -37.594 \\
\hline YWHAZ & MK2206 & -32.715 \\
\hline YWHAZ & Corannulene & -23.241 \\
\hline
\end{tabular}

Surprisingly, docetaxel outperformed BV02_9 in binding to the amphipathic groove of 14-3-3 proteins with $\Delta G$ values ranging from $-45.0 \mathrm{kcal} / \mathrm{mol}$ (YWHAB) to $-62.6 \mathrm{kcal} / \mathrm{mol}$ (YWHAZ) (Fig. 1e, Table 1). Consistent with expectations corannulene is a poor binder of 14-3-3 protein family members with $\Delta \mathrm{G}$ ranging from $-18.0 \mathrm{kcal} / \mathrm{mol}$ (YWHAE) to $-29.7 \mathrm{kcal} / \mathrm{mol}$ (YWHAH). In summary, our docking results show that BV02 binds to a range of 14-3-3 protein family members.

\section{Perturbation of PCa cells with BV02: cell viability}

Based on our docking data we established that BV02 and BV02_9 bind all 14-3-3 protein family members. We hypothesized that BV02 will affect cell viability similar to previous siRNA knock-down studies of YWHAZ carried out in a metastatic PCa model in which a $50 \%$ reduction in cell viability was observed [8]. We carried out the perturbation in cell culture using metastatic $\mathrm{PCa}$ models termed LNCaP (clone FGC) and PC-3. Unexpectedly, our results show no statistically significant change in cell viability of BV02 treated $\mathrm{LNCaP}$ or $\mathrm{PC}-3$ cells compared to DMSO treated control (Additional file 1: Figure S3). Taken together, inhibition of 14-3-3 proteins using BV02 results in no statistically significant change in cell viability while knock-down of YWHAZ with siRNAs using PCa models results in a decrease in cell viability.

\section{Perturbation of PCa cells with BV02: proteome changes}

To establish that BV02 does alter the proteome of perturbed PCa models, we quantified the proteome following perturbation of $\mathrm{PCa}$ models with BV02. The experiment was carried out in triplicate for each vehicle-treated (DMSO) and BV02 treated condition. Cells were exposed to BV02 for $24 \mathrm{~h}$ and then harvested, the proteome isolated and digested using sequence-specific serine protease trypsin. Following digest, peptides were purified and analyzed by LC-MS/MS. Mass spectrometry data were annotated using Andromeda search engine and label-free quantification carried out using the MaxQuant software. For subsequent analysis, only proteins and an adjusted $p$-value below 0.05 were considered regulated.

\section{Data integration with 14-3-3 interactome}

BV02 binds to the amphipathic groove of 14-3-3 proteins and thus inhibits binding of other proteins via a consensus phosphorylated peptide motif. Our shotgun proteomics data detects many large changes in protein abundance as a consequence of BV02 treatment. To distinguish between direct interruption of proteinprotein interactions due to $\mathrm{BV} 02$ and indirect or secondary effects we integrated our data set with an 
affinity purification data set in which a 14-3-3 protein was used as bait and interacting proteins identified using LC-MS/MS (14-3-3-APMS, Fig. 2a) [9]. 68 proteins were common between all three datasets (14-3-3-APMS, highly regulated proteins in LNCaP and PC-3 cells upon BV02 treatment) (Fig. 2b). Analyzing these 68 proteins for protein-protein interactions in STRING-DB v10.5 revealed a tight protein-protein network. Upon further inspection two layers of interactors were identified: there are eleven primary proteins interacting directly with 14-3-3 protein family members (labelled in blue/purple, Fig. 2c) while the remaining proteins are only indirectly connected to $14-3-3$ protein family members (labelled in grey, Fig. 2c). Additional file 1: Table S2 lists all eleven proteins directly interacting with at least one 14-3-3 protein family member. Of interest are proteins involved in AKT / mTOR signalling, such as AKT substrate 1 (AKTS1), protein kinase C (PRKCA) and regulatory-associated protein of mTOR (RPTOR). Further, of these eleven proteins, only three are not involved in cell cycle progression: DEK, TSR1, and AKTS1. To find 14-3-3 proteins which directly bind to proteins involved in AKT / mTOR signal transduction and proteins involved in cell cycle progression gives additional support to the hypothesis that by targeting structural proteins multiple pathways might be inhibited. Of special note in this context is that cytoskeleton associated protein 5 (CKAP5, labelled in purple in Fig. 2c) has both direct protein-protein interactions between 14-3-3 protein family members and other proteins directly interacting with 14-3-3 proteins giving CKAP5 a unique position in this protein-protein network.

Although we could not establish any compelling evidence for the use of BV02 in our PCa models, other models might benefit from the drug. For example, BV02 (at the concentration used in our experiments) induced a $60 \%$ reduction in cell viability using chronic myeloid leukaemia [6].

\section{Conclusions}

Structure-based drug design was used to assess binding specificity of inhibitor BV02 and related compounds for all seven human 14-3-3 proteins, revealing promiscuous binding. Surprisingly, docetaxel was identified as a strong binder to the amphipathic binding groove of

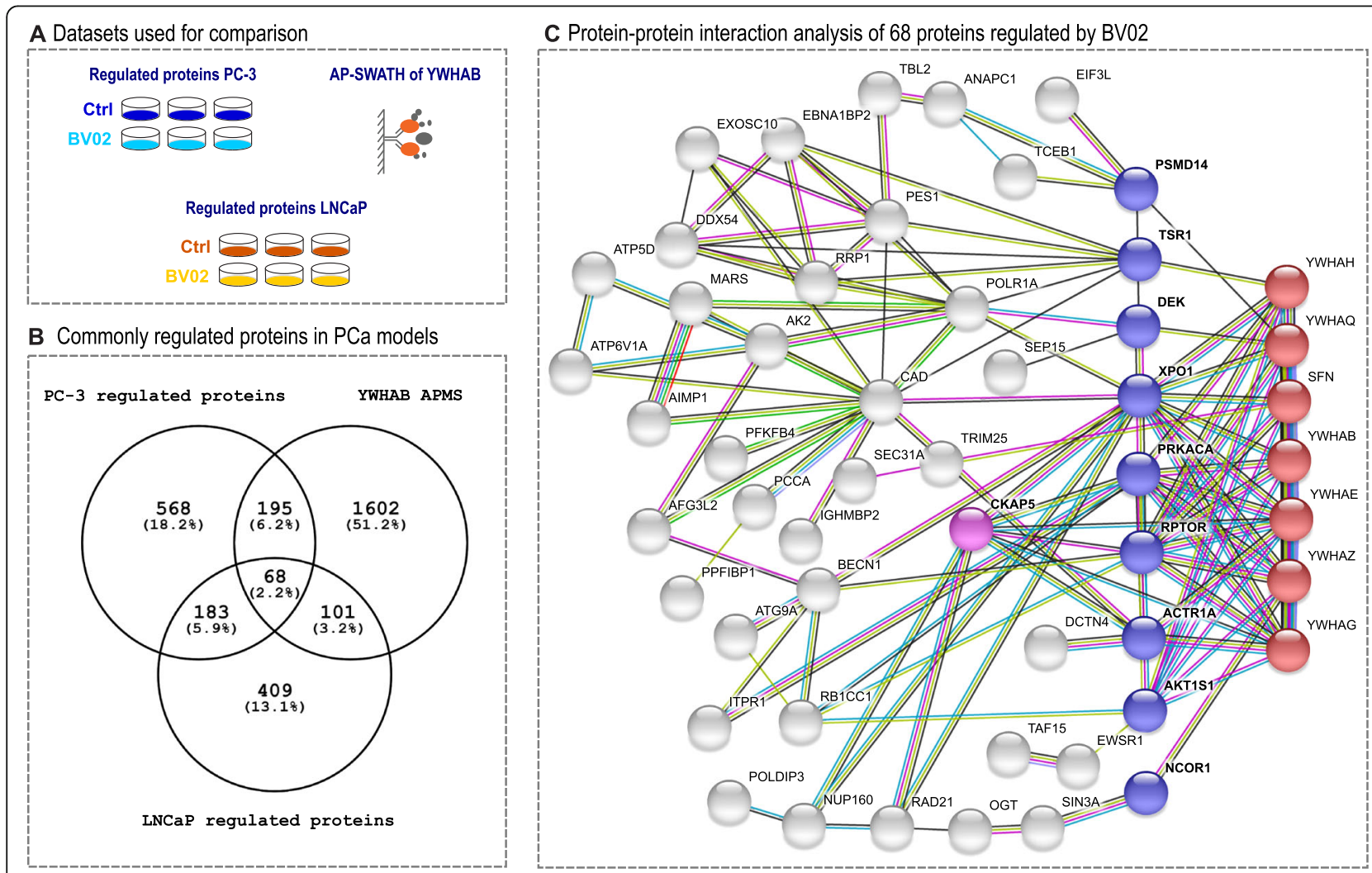

Fig. 2 Data sets integration. a Three data sets were used for data integration: LNCaP clone FGC and PC-3 cells perturbed with BV02 and highly regulated proteins integrated with the YWHAB interactome. b There are 68 proteins shared between all three proteomic data sets. c These 68 proteins form a tightly connected protein-protein interaction network (STRING-DB v10.5). 14-3-3 proteins shown with red dots directly interact with ten proteins (in blue and pink), while all other regulated proteins do not directly interact with 14-3-3 proteins 
14-3-3 proteins, especially for YWHAZ. In vivo experiments on PCa models demonstrate that BV02 does modulate direct protein binders of 14-3-3 proteins. However, modulation of binding using BV02 and downregulation of 14-3-3 proteins using siRNAs have different effects in PCa models.

\section{Additional files}

Additional file 1: Containing detailed description of materials and methods, modeling, supplementary tables and supplementary figures, (DOC $2230 \mathrm{~kb})$

\section{Abbreviations}

AKTS1: AKT substrate 1; CKAP5: cytoskeleton associated protein 5; CRPC: castrateresistant prostate cancer; ESCRT: Endosomal Sorting Complex Required For Transport; LC-MS/MS: Liquid chromatography coupled to mass spectrometer; MCRPC: Metastatic castrate-resistant prostate cancer; PCa: prostate cancer; PRKCA: protein kinase C; RPTOR: regulatory-associated protein of mTOR

\section{Acknowledgments}

We are grateful to the Chodera Lab and Tri-institutional Drug Discovery Institute for providing training and access to the Schrodinger Software Suite.

\section{Funding}

U.S. National Cancer Institute (NCI) P30 Cancer Center Support Grant (CCSG) P30

CA008748 to A.R. The funding covers general support for the research center.

\section{Availability of data and materials}

Raw mass spectrometry instrument files as well as parameter files in xml format and output files of MaxQuant software are available in MassIVE ID ftp://massive.ucsd.edu/MSV000081938.

\section{Authors' contributions}

$A R$ and HAE conceived and designed the article; AR and HAE wrote the paper. AR performed the SBDD analysis. HAE and AB performed cell culture, drug perturbations, and mass spectrometry experiments. All authors critically revised the manuscript. All authors read and approved the final manuscript.

\section{Ethics approval and consent to participate}

No humans were actively recruited to the work presented here.

\section{Consent for publication}

No humans were actively recruited to the work presented here.

\section{Competing interests}

The authors declare that they have no competing interests.

\section{Publisher's Note}

Springer Nature remains neutral with regard to jurisdictional claims in published maps and institutional affiliations.

\section{Author details}

${ }^{1}$ Molecular Biology Program, Memorial Sloan Kettering Cancer Center, New York, NY, USA. ${ }^{2}$ Systems Biology Ireland, University College Dublin, Belfield, Dublin 4, Ireland.

Received: 22 May 2018 Accepted: 15 October 2018

Published online: 31 October 2018

\section{References}

1. Gillessen S, Omlin A, Attard G, de Bono JS, Efstathiou E, Fizazi K, Halabi S, Nelson PS, Sartor O, Smith MR, et al. Management of patients with advanced prostate cancer: recommendations of the St Gallen advanced prostate Cancer consensus conference (APCCC) 2015. Ann Oncol. 2015; 26(8):1589-604.
2. Siegel RL, Miller KD, Jemal A. Cancer statistics, 2018. CA Cancer J Clin. 2018:68(1):7-30.

3. Root A, Ebhardt HA. A two-drug combination simulation study for metastatic castrate resistant prostate cancer. Prostate. 2018:78(15):1196-200.

4. Ebhardt HA, Root A, Liu Y, Gauthier NP, Sander C, Aebersold R. Systems pharmacology using mass spectrometry identifies critical response nodes in prostate cancer. NPJ Syst Biol Appl. 2018;4:26.

5. Sluchanko NN, Gusev NB. Moonlighting chaperone-like activity of the universal regulatory 14-3-3 proteins. FEBS J. 2017;284(9):1279-95.

6. Mancini M, Corradi V, Petta S, Barbieri E, Manetti F, Botta M, Santucci MA. A new nonpeptidic inhibitor of 14-3-3 induces apoptotic cell death in chronic myeloid leukemia sensitive or resistant to imatinib. J Pharmacol Exp Ther. 2011;336(3):596-604

7. Valensin D, Cau Y, Calandro P, Vignaroli G, Dello lacono L, Chiariello M, Mori $\mathrm{M}$, Botta M. Molecular insights to the bioactive form of BV02, a reference inhibitor of 14-3-3sigma protein-protein interactions. Bioorg Med Chem Lett. 2016;26(3):894-8.

8. Menon R, Deng M, Ruenauver K, Queisser A, Peifer M, Offermann A, Boehm $D$, Vogel W, Scheble V, Fend F, et al. Somatic copy number alterations by whole-exome sequencing implicates YWHAZ and PTK2 in castrationresistant prostate cancer. J Pathol. 2013;231(4):505-16.

9. Collins BC, Gillet LC, Rosenberger G, Rost HL, Vichalkovski A, Gstaiger M, Aebersold R. Quantifying protein interaction dynamics by SWATH mass spectrometry: application to the 14-3-3 system. Nat Methods. 2013;10(12):1246-53.
Ready to submit your research? Choose BMC and benefit from:

- fast, convenient online submission

- thorough peer review by experienced researchers in your field

- rapid publication on acceptance

- support for research data, including large and complex data types

- gold Open Access which fosters wider collaboration and increased citations

- maximum visibility for your research: over $100 \mathrm{M}$ website views per year

At $\mathrm{BMC}$, research is always in progress.

Learn more biomedcentral.com/submissions 